\title{
Novel RS1 mutations associated with X-linked juvenile retinoschisis
}

\author{
JUNHUI YI ${ }^{1,2}$, SHIQIANG $\mathrm{LI}^{2}$, XIAOYUN JIA ${ }^{2}$, XUESHAN XIAO ${ }^{2}$, PANFENG WANG $^{2}$, \\ XIANGMING GUO ${ }^{2}$ and QINGJIONG ZHANG ${ }^{2}$ \\ ${ }^{1}$ Department of Ophthalmology, The Third Xiangya Hospital, Central-South University, Changsha 410013; \\ ${ }^{2}$ State Key Laboratory of Ophthalmology, Zhongshan Ophthalmic Center, \\ Sun Yat-Sen University, Guangzhou 510060, P.R. China
}

Received November 6, 2011; Accepted December 15, 2011

DOI: $10.3892 /$ ijmm.2012.882

\begin{abstract}
To identify mutations in the retinoschisin (RS1) gene in families with X-linked retinoschisis (XLRS). Twenty families with XLRS were enrolled in this study. All six coding exons and adjacent intronic regions of $R S 1$ were amplified by polymerase chain reaction (PCR). The nucleotide sequences of the amplicons were determined by Sanger sequencing. Ten hemizygous mutations in $R S 1$ were detected in patients from 14 of the 20 families. Four of the ten mutations were novel, including c:176G >A (p:Cys59Tyr) in exon 3, c:531T >G (p:Tyr177X), c:607C $>$ G (p:Pro203Ala) and c:668G $>A$ (p:Cys223Tyr) in exon 6 . These four novel mutations were not present in 176 normal individuals. The remaining six were recurrent mutations, including c:214G >A (p:Glu72Lys), c:304C >T (p:Arg102Trp), c:436G >A (p:Glu146Lys), c:544C>T (p:Arg182Cys), c:599G>A (p:Arg200His) and c:644A>T (p:Glu215Val). Our study expanded the mutation spectrum of $R S 1$ and enriches our understanding of the molecular basis of XLRS.
\end{abstract}

\section{Introduction}

X-linked retinoschisis (XLRS, MIM 312700) is a hereditary retinal disease characterized by a splitting of the neurosensory retina, with a prevalence of 1:5,000 to 1:25,000 males worldwide (1). Typical fundus changes include radiating cysteic maculopathy in most cases and peripheral retinoschisis in half of the cases (2). However, the disease has a high degree of phenotypic variability (3-6), in which genetic testing is of value in confirming the diagnosis (4).

XLRS accounts for most congenital retinoschisis $(2,7)$ and is due to mutations in the retinoschisin gene (RSI, OMIM

Correspondence to: Dr Qingjiong Zhang, State Key Laboratory of Ophthalmology, Zhongshan Ophthalmic Center, Sun Yat-Sen University, 54 Xianlie Road, Guangzhou 510060, P.R. China E-mail: qingjiongzhang@yahoo.com

Key words: mutations, $R S 1$ gene, retinoschisis
$312700)$ localized on Xp22.13 $(8,9)$. The encoded protein, retinoschisin, is secreted from photoreceptors and bipolar cells as a functional homo-octameric complex that is thought to play a role in cellular adhesion and cell-to-cell interaction (10).

Gene transference to mouse models of X-linked juvenile retinoschisis, which suggest gene replacement may be a possible future therapy for patients (11-13). Genetic diagnosis is the basis for gene transference in the future. Therefore, we have to fully understand the molecular basis of XLRS. To date, more than 160 different $R S 1$ mutations have been identified in patients with XLRS (http://www.dmd.nl/rs), including small intragenic deletions, nonsense and missense mutations, frame shift insertions and deletions, and splice site mutations. However, there are still some RSI mutations that remain unknown.

In this study, we analyzed the coding exons and the adjacent regions of $R S 1$ in patients from 20 unrelated Chinese families with XLRS. Ten hemizygous mutations, including 4 novel mutations, were detected in 14 families.

\section{Subjects and methods}

Probands with XLRS from 20 unrelated families were enrolled in this study. Written informed consent was obtained from the participating individuals or their guardians prior to the collection of clinical data and genomic samples. This study was approved by the Internal Review Board of the Zhongshan Ophthalmic Center.

Mutation detection. Genomic DNA was prepared from venous leukocytes. Six pairs of primers (Table I) were used to amplify the six coding exons and the adjacent intronic sequence of RS1 (NCBI human genome build 37.2, NG_008659.1 for genomic DNA, NM_000330.3 for mRNA, and NP_000321.1 for protein). Touchdown polymerase chain reaction (PCR) was performed with decreasing $0.5^{\circ} \mathrm{C}$ per cycle from $64^{\circ} \mathrm{C}$ for the first 15 cycles then down to $57^{\circ} \mathrm{C}$ (the annealing temperature) for the remaining 21 cycles. GC buffer was used. DNA sequences of the amplicons were identified with ABI BigDye Terminator cycle sequencing kit version 3.1 (Applied Biosystems, Foster City, CA) on an ABI 3130 Genetic Analyzer (Applied Biosystems). Sequencing results 
Table I. Primers used for the amplification and sequencing of RS1.

\begin{tabular}{|c|c|c|c|c|}
\hline Exon & Direction & Primer sequence $\left(5^{\prime}-3{ }^{\prime}\right)$ & $\begin{array}{l}\text { Size of amplified } \\
\text { fragment (bp) }\end{array}$ & $\begin{array}{c}\text { Annealing } \\
\text { temperature }\left({ }^{\circ} \mathrm{C}\right)\end{array}$ \\
\hline 1 & $\begin{array}{l}\mathrm{F} \\
\mathrm{R}\end{array}$ & $\begin{array}{l}\text { GGTTAACTTGATGGGGCTCA } \\
\text { AACTGGAAAGCCATCCACAC }\end{array}$ & 374 & 57 \\
\hline 2 & $\begin{array}{l}\mathrm{F} \\
\mathrm{R}\end{array}$ & $\begin{array}{l}\text { TCTATTTCACTTTTCCATGTAACGA } \\
\text { ACCATGCCCAGCCAAAATA }\end{array}$ & 243 & 57 \\
\hline 3 & $\begin{array}{l}\mathrm{F} \\
\mathrm{R}\end{array}$ & $\begin{array}{l}\text { GACGATGCATAAGGACTGAGTG } \\
\text { AGCGTTCAGGGGGTTAATTC }\end{array}$ & 296 & 57 \\
\hline 4 & $\begin{array}{l}\mathrm{F} \\
\mathrm{R}\end{array}$ & $\begin{array}{l}\text { GCAAAGCAGATGGGTTTGTT } \\
\text { CCACCACGCCAGTTAATTTT }\end{array}$ & 359 & 57 \\
\hline 5 & $\begin{array}{l}\mathrm{F} \\
\mathrm{R}\end{array}$ & $\begin{array}{l}\text { CAGGGGGCTCTTTGGATG } \\
\text { ACAGAGGGCAGTGACAGGAG }\end{array}$ & 389 & 57 \\
\hline 6 & $\begin{array}{l}\mathrm{F} \\
\mathrm{R}\end{array}$ & $\begin{array}{l}\text { CACCCGCAAACTGCTTTAAC } \\
\text { TGCGAAATATAGCCCTGTCC }\end{array}$ & 384 & 57 \\
\hline
\end{tabular}

GC buffer was used in all amplifications. F, indicates the forward sequence; R, indicates the reverse sequence.

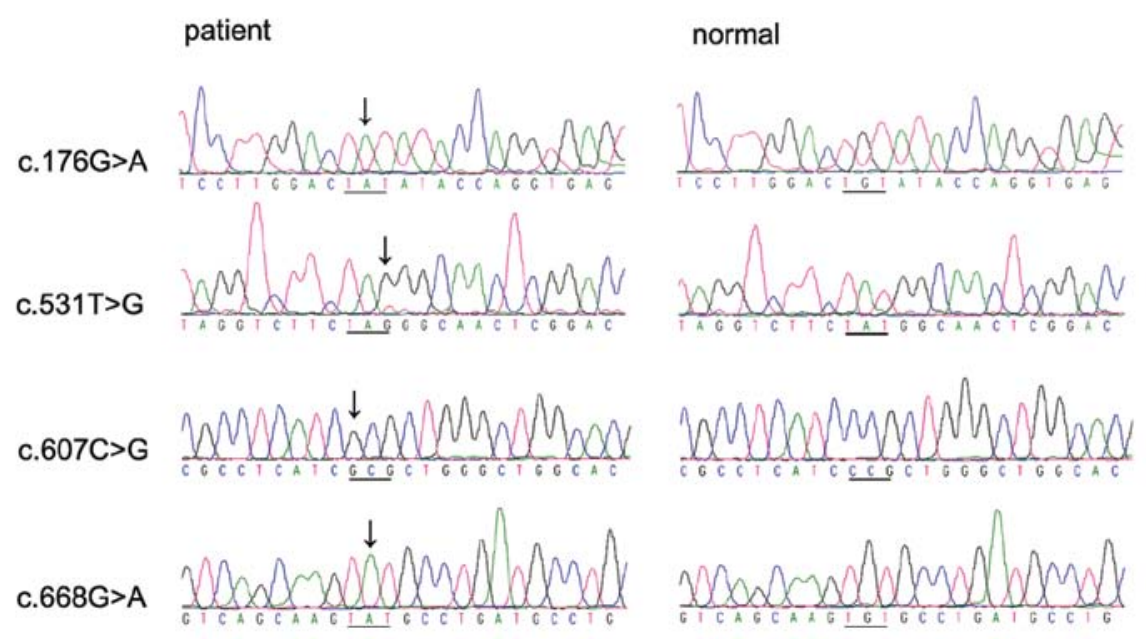

Figure 1. Sequence chromatography. Four novel sequence changes detected in the probands with RS are shown (left column) compared with corresponding normal sequences (right column).
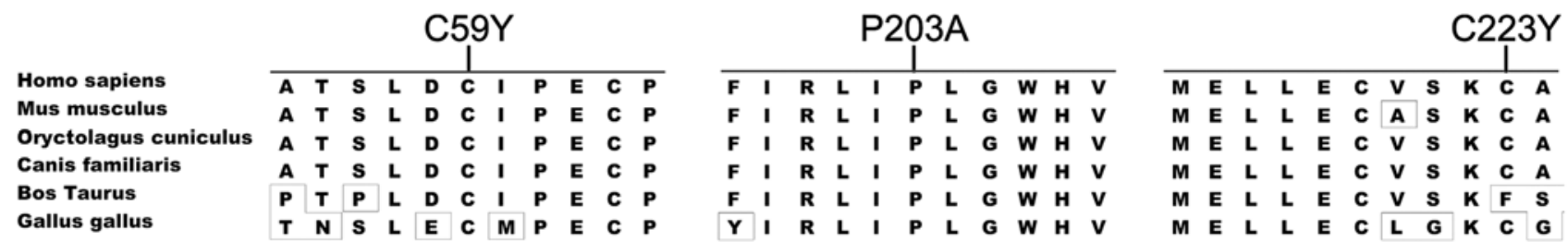

Figure 2. Protein sequence alignment of six RS1 orthologs. The regions with the novel p.C59Y and p.P203A mutations are highly conserved, C223Y is comparatively conserved.

and consensus sequences from the NCBI human genome database were compared by using the SeqMan II program of the Lasergene package (DNA Star, Inc., Madison, WI) and then aligned to identify variations. Each variation was confirmed by bidirectional sequencing. Mutation description followed the recommendation of the Human Genomic Variation Society (HGVS). Variations detected in patients were further evaluated in controls by sequencing 176 normal individuals.

The Sorting Intolerant From Tolerant (SIFT) program and the Polymorphism Phenotyping (PolyPhen-2) were used to 

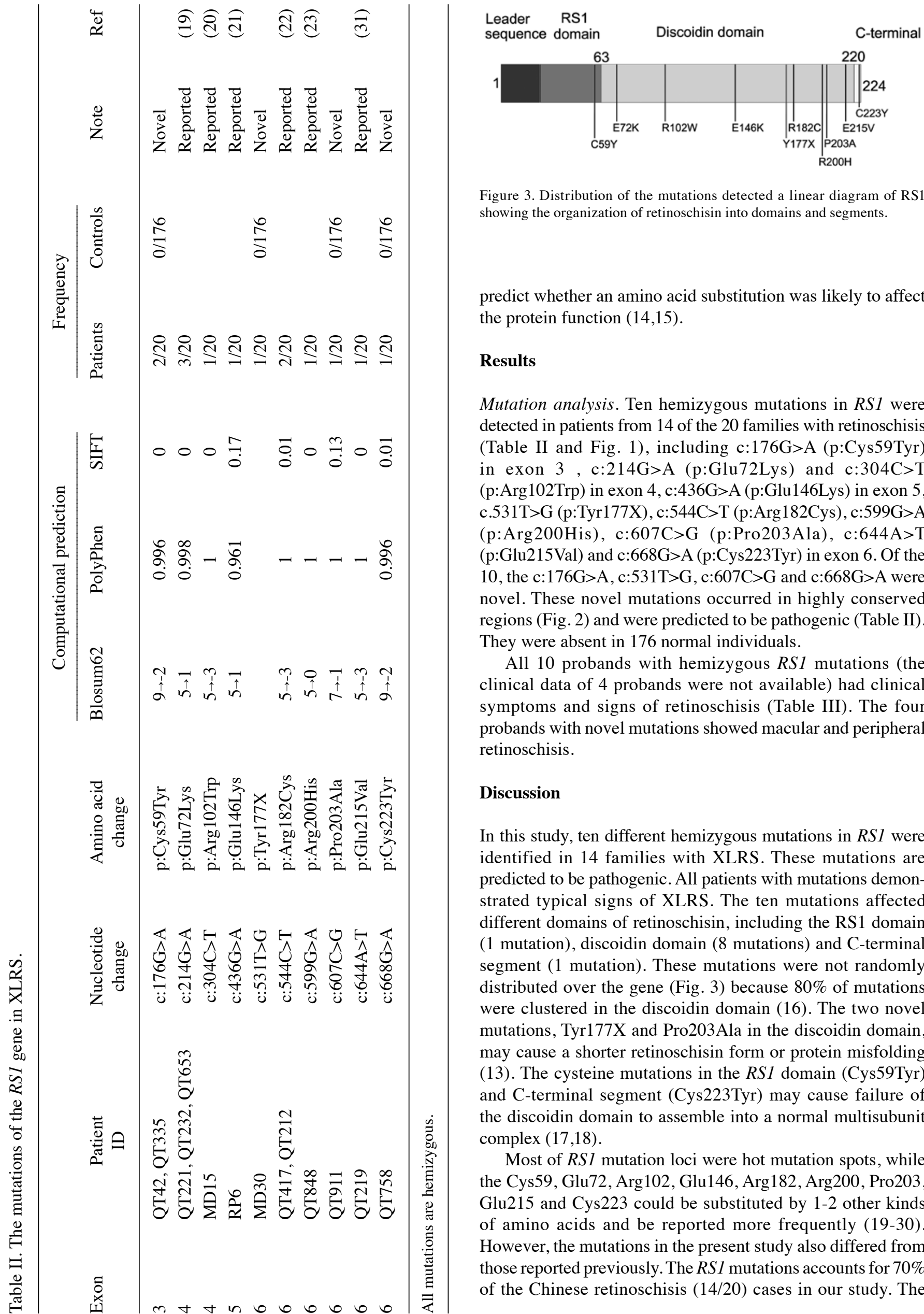

Figure 3. Distribution of the mutations detected a linear diagram of RS1 showing the organization of retinoschisin into domains and segments.

predict whether an amino acid substitution was likely to affect the protein function $(14,15)$.

\section{Results}

Mutation analysis. Ten hemizygous mutations in RS1 were detected in patients from 14 of the 20 families with retinoschisis (Table II and Fig. 1), including c:176G $>$ A (p:Cys59Tyr) in exon $3, c: 214 \mathrm{G}>\mathrm{A}$ (p:Glu72Lys) and $\mathrm{c}: 304 \mathrm{C}>\mathrm{T}$ (p:Arg 102Trp) in exon 4, c:436G >A (p:Glu146Lys) in exon 5, c.531T>G (p:Tyr177X), c:544C > T (p:Arg182Cys), c:599G >A (p:Arg200His), c:607C $>$ G (p:Pro203Ala), c:644A $>$ T (p:Glu215Val) and c:668G $>$ A (p:Cys223Tyr) in exon 6. Of the 10 , the $\mathrm{c}: 176 \mathrm{G}>\mathrm{A}, \mathrm{c}: 531 \mathrm{~T}>\mathrm{G}, \mathrm{c}: 607 \mathrm{C}>\mathrm{G}$ and $\mathrm{c}: 668 \mathrm{G}>\mathrm{A}$ were novel. These novel mutations occurred in highly conserved regions (Fig. 2) and were predicted to be pathogenic (Table II). They were absent in 176 normal individuals.

All 10 probands with hemizygous $R S 1$ mutations (the clinical data of 4 probands were not available) had clinical symptoms and signs of retinoschisis (Table III). The four probands with novel mutations showed macular and peripheral retinoschisis.

\section{Discussion}

In this study, ten different hemizygous mutations in $R S 1$ were identified in 14 families with XLRS. These mutations are predicted to be pathogenic. All patients with mutations demonstrated typical signs of XLRS. The ten mutations affected different domains of retinoschisin, including the RS1 domain (1 mutation), discoidin domain (8 mutations) and C-terminal segment (1 mutation). These mutations were not randomly distributed over the gene (Fig. 3) because $80 \%$ of mutations were clustered in the discoidin domain (16). The two novel mutations, Tyr177X and Pro203Ala in the discoidin domain, may cause a shorter retinoschisin form or protein misfolding (13). The cysteine mutations in the RSI domain (Cys59Tyr) and C-terminal segment (Cys223Tyr) may cause failure of the discoidin domain to assemble into a normal multisubunit complex $(17,18)$.

Most of $R S 1$ mutation loci were hot mutation spots, while the Cys59, Glu72, Arg102, Glu146, Arg 182, Arg200, Pro203, Glu215 and Cys 223 could be substituted by 1-2 other kinds of amino acids and be reported more frequently (19-30). However, the mutations in the present study also differed from those reported previously. The RS1 mutations accounts for $70 \%$ of the Chinese retinoschisis (14/20) cases in our study. The 


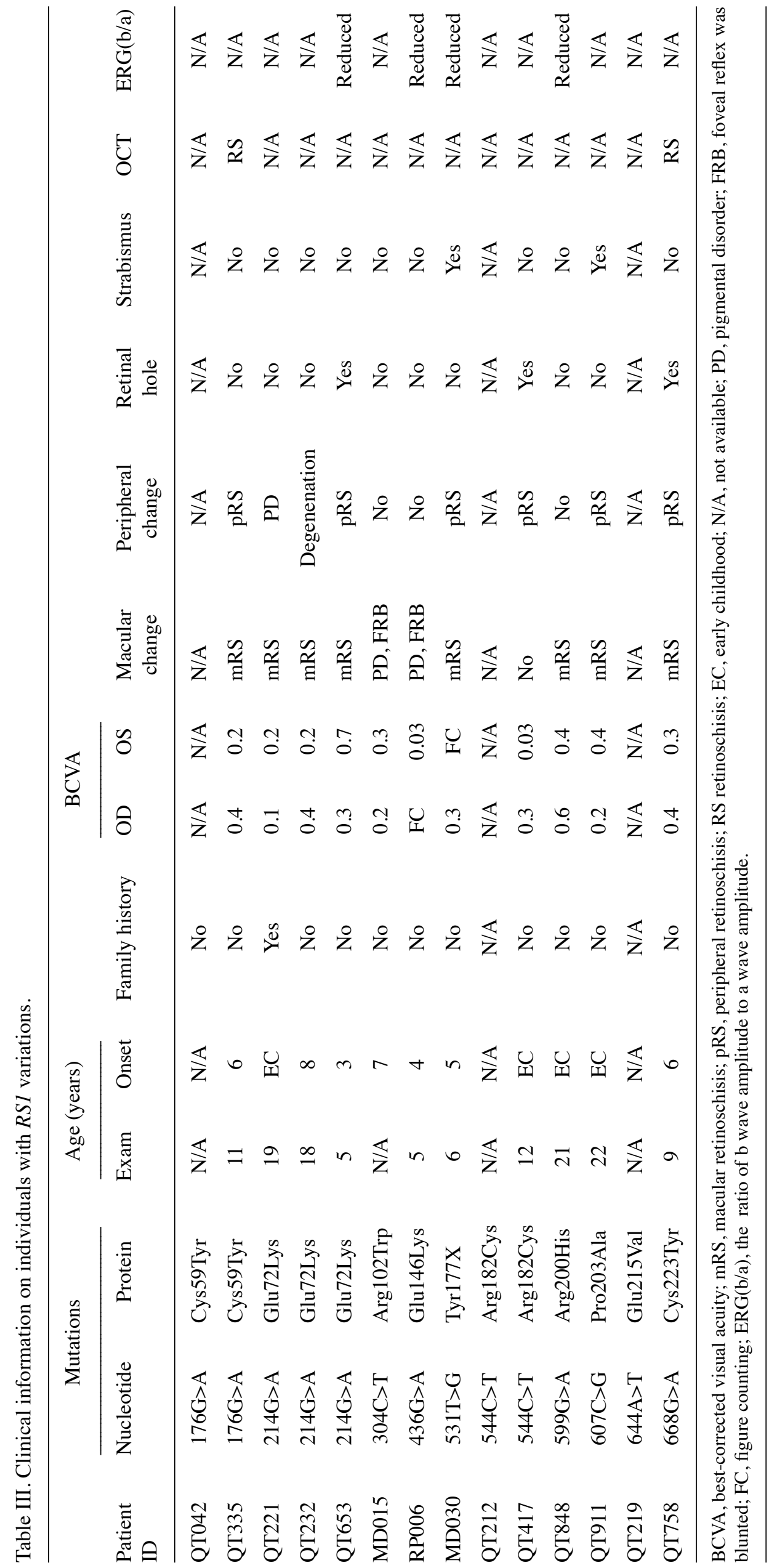


Cys59Tyr, Tyr177X, Pro203Ala, Glu215Val and Cys223Tyr mutations only are present in the Chinese population (31), and the Cys59Tyr mutation was more common (10\% frequency in our retinoschisis cases). The Glu72Lys mutation is the most common among Chinese (15\%) as well as other populations $(19,32)$, while another very common mutation, Pro192Ser (33), which was reported from people of different ethnic backgrounds was not found. We do not know whether the spectrum and frequency of $R S 1$ gene in the Chinese is different from others. Our study contributes to the current state of knowledge.

In summary, we identified ten mutations in 14 of 20 families with XLRS. Our results expand the mutation spectrum of RS1 that might enrich our understanding of the molecular basis of XLRS in the Chinese population.

\section{Acknowledgements}

The authors thank all of the patients and controls subjects for their participation. This study was supported by the Open Research Fund Program of State Key Laboratory of Ophthalmology, Zhongshan Ophthalmic Center, Sun Yat-Sen University, and in part by grant 30725044 from the National Science Fund for Distinguished Young Scholars.

\section{References}

1. MacDonald IM and Sasi R: Molecular genetics of inherited eye disorders. Clin Invest Med 17: 474-498, 1994.

2. Sikkink SK, Biswas S, Parry NR, Stanga PE and Trump D: X-linked retinoschisis: an update. J Med Genet 44: 225-232, 2007.

3. Apushkin MA, Fishman GA and Rajagopalan AS: Fundus findings and longitudinal study of visual acuity loss in patients with X-linked retinoschisis. Retina 25: 612-618, 2005.

4. Tantri A, Vrabec TR, Cu-Unjieng A, Frost A, Annesley WH Jr and Donoso LA: X-linked retinoschisis: a clinical and molecular genetic review. Surv Ophthalmol 49: 214-230, 2004.

5. Shukla D, Rajendran A, Gibbs D, Suganthalakshmi B, Zhang K and Sundaresan P: Unusual manifestations of X-linked retinoschisis: clinical profile and diagnostic evaluation. Am J Ophthalmol 144: 419-423, 2007.

6. Kim JE, Ruttum MS, Koeberl MJ, Hassemer EL and Sidjanin DJ: Genetic and clinical evaluation of juvenile retinoschisis. J AAPOS 13: 215-217, 2009.

7. Sauer CG, Gehrig A, Warneke-Wittstock R, et al: Positional cloning of the gene associated with X-linked juvenile retinoschisis. Nat Genet 17: 164-170, 1997.

8. Mendoza-Londono R, Hiriyanna KT, Bingham EL, et al: A Colombian family with X-linked juvenile retinoschisis with three affected females finding of a frameshift mutation. Ophthalmic Genet 20: 37-43, 1999.

9. Huopaniemi L, Rantala A, Tahvanainen E, de la Chapelle A and Alitalo T: Linkage disequilibrium and physical mapping of X-linked juvenile retinoschisis. Am J Hum Genet 60: 1139-1149, 1997.

10. Besch D and Rudolph G: Genetic diseases of the eye. Klin Monbl Augenheilkd 222: 955-971, 2005 (In German).

11. Min SH, Molday LL, Seeliger MW, et al: Prolonged recovery of retinal structure/function after gene therapy in an Rs1h-deficient mouse model of X-linked juvenile retinoschisis. Mol Ther 12: 644-651, 2005

12. Dyka FM and Molday RS: Coexpression and interaction of wild-type and missense RS1 mutants associated with X-linked retinoschisis: its relevance to gene therapy. Invest Ophthalmol Vis Sci 48: 2491-2497, 2007.
13. Molday RS: Focus on molecules: retinoschisin (RS1). Exp Eye Res 84: 227-228, 2007.

14. Ng PC and Henikoff S: Predicting deleterious amino acid substitutions. Genome Res 11: 863-874, 2001.

15. Sunyaev S, Ramensky V, Koch I, Lathe W III, Kondrashov AS and Bork P: Prediction of deleterious human alleles. Hum Mol Genet 10: 591-597, 2001.

16. Molday LL, Hicks D, Sauer CG, Weber BH and Molday RS: Expression of X-linked retinoschisis protein RS1 in photoreceptor and bipolar cells. Invest Ophthalmol Vis Sci 42: 816-825, 2001.

17. Wu WW, Wong JP, Kast $\mathbf{J}$ and Molday RS: RS1, a discoidin domain-containing retinal cell adhesion protein associated with $\mathrm{X}$-linked retinoschisis, exists as a novel disulfide-linked octamer. J Biol Chem 280: 10721-10730, 2005.

18. Wu WW and Molday RS: Defective discoidin domain structure, subunit assembly, and endoplasmic reticulum processing of retinoschisin are primary mechanisms responsible for X-linked retinoschisis J Biol Chem 278: 28139-28146, 2003.

19. Hotta Y, Fujiki K, Hayakawa M, et al: Japanese juvenile retinoschisis is caused by mutations of the XLRS1 gene. Hum Genet 103: 142-144, 1998.

20. Dodds JA, Srivastava AK and Holden KR: Unusual phenotypic expression of an XLRS1 mutation in X-linked juvenile retinoschisis. J Child Neurol 21: 331-333, 2006.

21. Khan NW, Jamison JA, Kemp JA and Sieving PA: Analysis of photoreceptor function and inner retinal activity in juvenile X-linked retinoschisis. Vision Res 41: 3931-3942, 2001.

22. Mashima Y, Shinoda K, Ishida S, et al: Identification of four novel mutations of the XLRS1 gene in Japanese patients with X-linked juvenile retinoschisis. Mutation in brief no. 234. Online. Hum Mutat 13: 338, 1999.

23. Lledo B, Ten J, Rodriguez-Arnedo D, Llacer J and Bernabeu R: Preimplantation genetic diagnosis of X-linked retinoschisis. Reprod Biomed Online 16: 886-892, 2008.

24. Tuvdendorj D, Isashiki Y, Ohba N, Sonoda S and Izumo S: Two Japanese patients with mutations in the XLRS1 gene. Retina 22: 354-357, 2002.

25. Inoue $\mathrm{Y}$, Yamamoto $\mathrm{S}$, Inoue $\mathrm{T}$, et al: Two novel point mutations of the XLRS1 gene in patients with X-linked juvenile retinoschisis. Am J Ophthalmol 134: 622-624, 2002.

26. Simonelli F, Cennamo G, Ziviello C, et al: Clinical features of $\mathrm{X}$ linked juvenile retinoschisis associated with new mutations in the XLRS1 gene in Italian families. Br J Ophthalmol 87: 1130-1134, 2003.

27. Walia S, Fishman GA, Molday RS, et al: Relation of response to treatment with dorzolamide in X-linked retinoschisis to the mechanism of functional loss in retinoschisin. Am J Ophthalmol 147: 111-115, 2009.

28. Wang T, Zhou A, Waters CT, O'Connor E, Read RJ and Trump D: Molecular pathology of $\mathrm{X}$ linked retinoschisis: mutations interfere with retinoschisin secretion and oligomerisation. Br J Ophthalmol 90: 81-86, 2006

29. Dyka FM, Wu WW, Pfeifer TA, Molday LL, Grigliatti TA and Molday RS: Characterization and purification of the discoidin domain-containing protein retinoschisin and its interaction with galactose. Biochemistry 47: 9098-9106, 2008.

30. Ma X, Li X and Wang L: Novel XLRS1 gene mutations cause $\mathrm{X}$-linked juvenile retinoschisis in Chinese families. Jpn J Ophthalmol 52: 48-51, 2008.

31. Zeng M, Yi C, Guo X, et al: Identification of novel mutations in the XLRS1 gene in Chinese patients with X-linked juvenile retinoschisis. Curr Eye Res 32: 685-691, 2007.

32. Lesch B, Szabo V, Kanya M, et al: Clinical and genetic findings in Hungarian patients with X-linked juvenile retinoschisis. Mol Vis 14: 2321-2332, 2008.

33. Eksandh LC, Ponjavic V, Ayyagari R, et al: Phenotypic expression of juvenile X-linked retinoschisis in Swedish families with different mutations in the XLRS1 gene. Arch Ophthalmol 118: 1098-1104, 2000. 